BULLETIN Bulletin hispanique

HispaniquE Université Michel de Montaigne Bordeaux

116-2 | 2014

Référentialité/autoréférentialité dans le roman espagnol contemporain : bilan et perspectives

\title{
Référence et autoréférence
}

deux autoportraits générationnels de Juan Marsé

Jean Tena

\section{(2) OpenEdition}

Journals

\section{Édition électronique}

URL : http://journals.openedition.org/bulletinhispanique/3576

DOI : 10.4000/bulletinhispanique.3576

ISBN : 979-10-300-0156-3

ISSN : $1775-3821$

Éditeur

Presses universitaires de Bordeaux

\section{Édition imprimée}

Date de publication : 1 décembre 2014

Pagination : 805-811

ISBN : 978-2-86781-963-6

ISSN : 0007-4640

Référence électronique

Jean Tena, «Référence et autoréférence », Bulletin hispanique [En ligne], 116-2 | 2014, mis en ligne le 05 mars 2015, consulté le 01 mai 2019. URL : http://journals.openedition.org/bulletinhispanique/3576 ;

DOI : 10.4000/bulletinhispanique.3576 


\title{
Référence et autoréférence : deux autoportraits générationnels de Juan Marsé
}

\author{
Jean Tena \\ Université Paul Valéry - Montpellier 3
}

Dans les deux séries de portraits littéraires intitulées Señoras y señores (1975 et 1988), Juan Marsé propose autant d'autoportraits individuels mais aussi générationnels par le biais de deux leitmotivs caractéristiques de la "génération innocente " : la dénonciation de l'escamotage de la réalité par le franquisme; le rôle fondamental de la mémoire dans la recherche du temps escamoté.

Mots-clés: Juan Marsé, franquisme, " génération innocente ", escamotage, mémoire.

En ambas series de retratos literarios tituladas Señoras y señores (1975 y 1988) Juan Marsé propone sendos autorretratos individuales pero también generacionales mediante dos leitmotivs característicos de la "generación inocente " : la denuncia del escamoteo de la realidad por el franquismo; el papel fundamental de la memoria en la busca del tiempo escamoteado.

Palabras claves: Juan Marsé, franquismo, "generación inocente», escamoteo, memoria.

In both series of literary portraits entitled Señoras y señores (1975 and 1988), Juan Marsé offers many individual as generational self-portraits based upon two typical leitmotivs of the "innocent generation»: the denunciation of franquoism for dodging reality and the prominent part of memory in the search of this dodged past time.

Keywords: Juan Marsé, franquoism, «innocent generation», sneaking, memory. 
"Vierte, expande / en tu memoria aquel confinamiento, / busca otra vez en las estribaciones / inciertas del pasado, vuelve / a ese tiempo de lividos contornos",

José Manuel Caballero Bonald, Las adivinaciones (1952). "[...] el hierro al rojo vivo de la memoria ", Jorge Semprún, Autobiografía de Federico Sánchez (1977). "[...] algunos por si acaso, aún mantenemos el dedo en el gatillo de la memoria", Juan Marsé, Un día volveré (1982). " [...] un texto de reconocimiento generacional», Carlos Barral, Penúltimos castigos (1983). "[...] los valores de naftalina que nos habian impuesto", Oriol Regás, Los años divinos (2010).

Comme la plupart des écrivains, Juan Marsé introduit dans certaines de ses œuvres des éléments autobiographiques anecdotiques ou fondamentaux, plus ou moins manipulés en fonction de l'effet recherché.

Il ne s'agit parfois que d'une simple allusion, relativement distanciée, souvent ironique, comme celle qui a pour cadre une "sala de baile " où la protagoniste de Últimas tardes con Teresa essaie de se divertir. Brusquement, "notó en las nalgas un pellizco de maestro, muy lento, pulcro y aprovechado". Teresa, "roja como un tomate ", ne verra qu'" una silueta encorvada, los hombros escépticos $y$ encogidos de un tipo bajito que se escabullía riendo " et entendra une de ses voisines dénoncer l'agresseur : "se llama Marsé, es uno bajito, moreno, de pelo rizado, y siempre anda metiendo mano". Une sorte de Don Juan au petit pied, un "caradura " capable même de relancer ses victimes (" me dio su número de teléfono por si quería algo de él $»^{1}$ ).

C'est bien au-delà de l'anecdotique que se situe l'emploi récurrent du binôme Marés (anagramme transparente de Marsé)/Faneca. Il s'agit là d'un élément autobiographique fondamental. À cause de la mort de sa mère quinze jours après sa naissance et des problèmes d'ordre économique qui assaillent son père veuf, Juan, né Faneca, sera adopté par un couple sans enfants et deviendra Juan Marsé Carbó .

Ce binôme originel, ancré dans la personnalité de l'écrivain générera, par exemple, un éventail de possibilités spéculaires dans El amante bilingüe (1990).

Si les éléments autobiographiques peuvent être importants, voire essentiels, dans les œuvres de fiction, ils sont fondamentaux dans les deux autoportraits qu'on a choisi d'analyser. Ils figurent dans deux séries de "portraits littéraires " intitulés Señoras y señores. À partir d'une photographie de la personnalité choisie, Marsé développe ce que Manuel Vázquez Montalbán qualifie de

1. Juan Marsé, Últimas tardes con Teresa, Barcelona, Seix Barral, 1971 (cuarta edición), p. 256.

2. Fernando Valls détaille les circonstances de cette adoption, à laquelle il attribue « cierto hálito legendario ", dans son édition critique de Ronda del Guinardó, Barcelona, Crítica, 2005, p. 8.

3. La première série, publiée dans les années 70 par la revue Por favor est reprise dans un ouvrage édité en 1975 (Barcelona, Punch Ediciones) ; la deuxième (El País, mars-décembre 1987) paraît chez l'éditeur barcelonais Tusquets en 1988 . On les désignera par $S 1$ et $S 2$. 
"retrato literario, lectura en las rayas de los rostros y los gestos" $(S 1$, p. 6) ou encore de "pequeñas sintesis geniales» $(S 1, \mathrm{p} .7)$. Dans ces textes où le cinéma se taille la part du lion (ce qui n'est guère étonnant quand on connaît la cinéphilie boulimique de l'auteur) figurent un "Juan Marsé» (S1, p. 58, avec deux photos du personnage écolier et adulte ; cet autoportrait a été publié dans Por Favor le 25 octobre 1974) et un "Autorretrato" (S2, p. 173-174; la photo n'est pas reprise. Elle figure dans El Pais du 27 décembre 1987 et représente l'auteur déguisé en diable et photographié par son fils. Ce cliché, colorisé en rouge, illustre la couverture du livre). On laissera de côté les détails physiques proches des passages de Últimas tardes con Teresa évoqués plus haut (" estatura escasa, escépticos los hombros ", S1, p. 58 ; "escépticos los hombros", S2, p. 173 ; "El tipo es bajo, desmañado ", S2, p. 174) ou le portrait moral et intellectuel, voire littéraire ("su vieja disposición para la trola y el chisme y el vamos a contar mentiras tra-la-rá ", S1, p. 58). Tout cela pourrait faire l'objet d'une autre publication. On n'a retenu que deux éléments qui font de ces autoportraits ceux d'une génération qu'on a souvent qualifiée d' "innocente » : l'escamotage de la réalité par le franquisme et le rôle fondamental de la mémoire dans la recherche du temps escamoté.

À propos du premier point, la réaction de Marsé est sans ambages : la conséquence de "casi cuarenta años de censura " est "una adolescencia que le fue escamoteada" (S1, p. 58). Même son de cloche dans le portrait d'un autre membre de la "génération innocente", le cinéaste Carlos Saura : "la memoria que fue manipulada, camuflada y adulterada" (S1, p. 46). Le rythme ternaire renforcé par la triple rime en -ada confirme l'implacabilité du jugement. En quelques mots, Marsé plonge son lecteur au cœur de cette période sur laquelle un écrivain plus jeune revenait récemment : "El poder del régimen cambiando el sentido de las palabras. Los nombres de antes ya no sirven "; il n'y a qu'un passé, celui des vainqueurs : "El otro fue silenciado, tergiversado en el imaginario de la gente, prohibido en la imaginería impuesta por el nuevo régimen ${ }^{4}$.

Cette conscience aiguë de l'escamotage organisé par le franquisme triomphant est, sans doute, une des caractéristiques essentielles de cette génération. Après une expérience presque ludique de la guerre civile (Les longues vacances de 1936, selon le titre d'un film de Jaime Camino tourné en 1976) vient le temps des " années de pénitence " glosées par Carlos Barral en 1975. Les pouvoirs politique et familial vont imposer à ces " enfants de la guerre " une sorte de régénération morale qui passe par un véritable lavage de cerveau organisé, en particulier sur le plan de l'enseignement et de l'information.

Après bien d'autres, Carmen Martín Gaite rappelle, au passage, « que viviamos rodeados de ignorancia y represión" à cause, en particulier, de "aquellos deficientes libros de texto que bloquearon nuestra enseñanza "s. À quelques exceptions près

4. Alfons Cervera, «La memoria histórica en España : desde el silencio a la moda y el punto final » dans Georges Tyras et Juan Vila, Memoria y testimonio. Representaciones memorísticas en la España contemporánea, Madrid, Verbum, 2012, p. 247-258, p. 249 et 250.

5. Carmen Martín Gaite, El cuarto de atrás, Barcelona, Destino, 1978, p. 69. 
et à la suite d'une "depuración" qui a éliminé des milliers d'enseignants, l'éducation primaire et secondaire est assurée par de véritables spécialistes de la répression intellectuelle ("Aquellos hombres/predicaban miedo ") le plus souvent médiocres pédagogues.

Dans un premier temps, l'enseignement était mutilé, un certain nombre d'auteurs étant, tout simplement, interdits. Dans un deuxième temps, il était manipulé. Dans un ouvrage très documenté, Fernando Valls cite un certain nombre de ces manipulations où Kafka côtoie Ubu ${ }^{7}$. Histoire, grammaire, littérature, langues vivantes, tout est prétexte à exalter le "Glorioso Movimiento " ("Glorious Movement" en anglais) et à permettre aux petits Espagnols d'aller "por el Imperio hacia Dios» dans une Espagne "Una, grande y libre ".

Un seul exemple suffira pour montrer l'étendue de ces manipulations à répétition : le Catecismo patriótico español de Menéndez-Reigada déclaré manuel scolaire à partir du $1^{\mathrm{er}}$ mars $1939^{8}$. L'auteur, frère prêcheur et évêque, déclare, sans sourciller, que l'Espagne fut " colocada providencialmente por Dios en el centro del mundo" (p. 35) et que "la lengua castellana será la lengua de la civilización en lo futuro, porque el inglés y el francés, que con ella pudieran compartir esta función, son lenguas tan gastadas, que van camino de una disolución completa" (p. 40). Pour cet éminent linguiste, les autres langues péninsulaires ne sont que des dialectes caractérisés par «su pobreza lingüistica y filológica» (p. 40). A partir de telles bases "scientifiques ", on peut affirmer n'importe quoi : que le marxisme est fils du protestantisme (p. 48), que le tribunal de l'Inquisition a été " tan beneficioso " (p. 49), que seuls les "rouges " ont été aidés par des puissances étrangères "que volcaron su material bélico, su ayuda económica y sus hombres por cientos de miles en favor del gobierno de Valencia" (p. 57). On ne s'étonnera pas de découvrir que les ambitions colonisatrices de l'Espagne étaient " generosas, leales, desinteresadas" (p. 66) et que "el Estado totalitario cristiano no es contrario a la libertad humana, [...] la mejor garantía de la verdadera libertad, amparando al débil contra el fuerte, fomentando la virtud, la honradez, la justicia y el trabajo " (p. 79). Bien évidemment, grâce à la "Cruzada", l'Espagne est à nouveau le centre d'un monde "que nos contempla y admira" (p. 85) malgré les efforts de ses ennemis qui ne peuvent être que sept : "el liberalismo, la democracia, el judaísmo, la masonería, el capitalismo, el marxismo y el separatismo" (p. 86). La messe est dite et les écoliers espagnols prêts à devenir, selon Hilari Raguer, "los borregos de Franco" (p. 28). On comprend que, des dizaines d'années plus tard, un poète de cette génération sacrifiée peste contre " tantas y tan lóbregas alegorias de la falsedad/como me habian ido trasmitiendo a través de manuales breviarios catecismos $"$ ?.

6. José Agustín Goytisolo, "Mis maestros ", Claridad, 1961 (Poesía completa, Barcelona, Lumen, 2009, p. 97).

7. Fernando Valls, La enseñanza de la literatura en el franquismo (1936-1951), Barcelona, Antoni Bosch, 1983.

8. Menéndez-Reigada, Catecismo patriótico español, republié en 2003 (Barcelona, Península) avec un prologue démystificateur de Hilari Raguer.

9. José Manuel Caballero Bonald, Entreguerras, Barcelona, Seix Barral, 2012, p. 33. Le poète 
Après l'enseignement, la répression et la manipulation continuaient dans le cadre des activités scolaires et parascolaires. La mixité est supprimée, les enfants et les adolescents sont pris en main par le "Frente de Juventudes »... Mais, en dehors de la sphère scolaire ou universitaire, l'escamotage continue par l'intermédiaire de ce qu'on n'appelle pas encore les médias. Dans ce domaine, en particulier, la censure est omniprésente et implacable, la "consigna" sans appel ${ }^{10}$.

Manipulation et triomphalisme sont les deux piliers de la presse espagnole de l'après-guerre. Le sujet étant battu et rebattu depuis 1975, on se contentera d'évoquer quelques points relativement pittoresques. Par exemple la transcription journalistique du retournement d'alliances effectué par Franco vers la fin de la Deuxième Guerre Mondiale : des prophéties du Caudillo sur la défaite probable des Alliés (1941-1943) on passe à " l'objectivité » en ce qui concerne les succès "angloyanquis» tout en limitant l'écho des victoires soviétiques (1944). Tous les domaines, fondamentaux ou accessoires, sont concernés. Dans le cadre de ce qu'il qualifie de "rigurosa lobotomía del conocimiento histórico ", Román Gubern affirme que, en ce qui concerne les camps d'extermination nazis, «la mayor parte de los españoles ignoró el genocidio " jusqu'aux années 60. Sur le plan du pittoresque, le même auteur nous apprend que, dans les années 40 , face à l'avalanche de publicité médiatique, "los paños higiénicos para la menstruación no podian anunciarse $»^{11}$.

Comme l'enseignement et les médias, tous les domaines étaient touchés par cet escamotage. Le cas particulier du cinéma (si cher à Juan Marsé) est trop connu pour qu'il soit utile d'insister. Musique, danse, chanson, fêtes (suppression du Carnaval), vie quotidienne, sexualité : tout était soigneusement inspecté, censuré, stérilisé, éliminé le cas échéant. Il ne restait plus, selon Luis Carandell, que "pangelinguas, tantumergos, triduos, novenas, trisagios" ${ }^{12}$.

Pour la génération de Marsé, ce matraquage continu (surtout dans les années 40, celles, essentielles, de l'enfance et de l'adolescence) va provoquer, en premier lieu, une prise de conscience ("Mis ideas de la guerra cambiaron/después, mucho después/de que hubiera empezado la posguerra " ${ }^{13}$ ) puis un engagement, souvent dans les rangs du Parti communiste espagnol clandestin (comme Marsé pour une courte période parisienne dans les années 60). Sur le plan littéraire, cet engagement débouchera sur le réalisme social dont Tiempo de silencio (1962)

évoque aussi, p. 35, "una vaga conciencia confiscada".

10. On n'est pas loin de l'univers des anti-utopies littéraires (1984 d'Orwell ou Parábola del náufrago de Delibes) quand on lit les élucubrations d'un certain Prado y López (Ética y estética del periodismo español, 1943) : "La consigna es para los periódicos luz en el horizonte, señal de seguridad, guia oportuna. La consigna unifica también, salvando a todos del peligro del error. La censura de ahora no está inspirada por un criterio particular más o menos respetable, sino por el interés de España. Luego la Prensa española es hoy más libre que nunca ». C.Q.F.D ! (Cité par Fernando Díaz-Plaja, La España franquista en sus documentos, Barcelona, Plaza y Janés, 1976, p. 132).

11. Román Gubern, Viaje de ida, Barcelona, Anagrama, 1997, p. 308, 46 et 81.

12. Luis Carandell, El día más feliz de mi vida. Memorias, Madrid, Espasa, 2000, p. 250.

13. Jaime Gil de Biedma, «Intento formular mi experiencia de la guerra », Moralidades, 1966 (Las personas del verbo, Barcelona, Seix Barral,1982, p. 124). 
sonnera le glas. Les solutions de repli seront diverses mais l'une d'elles se trouvera privilégiée : le recours à la mémoire individuelle et collective, le seul moyen efficace de s'opposer au décervelage organisé par le franquisme. Il faut à tout prix récupérer, recréer le temps escamoté ("Por eso escribo esto [...]. Es una manera de oponer a la historia que ellos me cuentan la historia tal como ocurrió $\left.»^{14}\right)$.

Le premier autoportrait est déjà explicite sur ce point fondamental : «Mientras el país no sepa qué hacer con su pasado, jamás sabrá qué hacer con su futuro " (S1, p. 58). Et, comme auparavant, le portrait de Carlos Saura (un spécialiste du cinéma de la mémoire, un "memorión " ${ }^{15}$ dont même le physique "reposa en la memoria ") vient renforcer cette caractéristique de la génération : "recuperar la memoria perdida del pais, la memoria que fue manipulada, camuflada y adulterada"; " incansable bucear por el pasado que ha de explicarnos el presente"; "Sin memoria, no somos nada" (S1, p. 46). En 1987, douze ans après la mort de Franco, la mémoire, la nostalgie sont toujours présentes (S2, p. 173) ainsi que le rappel d'une ineffaçable "luctuosa telaraña franquista de casi cuarenta años" (S2, p. 174). Le "gatillo de la memoria " ${ }^{16}$ fonctionne toujours. Cette arme offensive et défensive (" chargée de passé " pour parodier le titre d'un poème célèbre de Gabriel Celaya mis en musique par Paco Ibáñez) va envahir les œuvres de Marsé et des membres de sa génération, engendrer une véritable "écriture de la mémoire » longuement évoquée dans une publication antérieure ce qui nous évitera de nous répéter ${ }^{17}$. Face à une attitude frileuse "que trata los recuerdos como flor de invernadero ", Carmen Martín Gaite conseille plutôt d'être "capaz de echarlos al río revuelto de la memoria ${ }^{18}$. Frustrée d'une part non négligeable de ses virtualités, la " génération innocente " n’aura donc pu devenir adulte et exorciser ses démons qu'en récupérant sa mémoire et celle de l'Espagne tout entière : "Ya soy mayor, ya soy memoria " se dit Sarnita, meneur de jeu du roman de Marsé Si te dicen que cai ${ }^{19}$.

14. Antonio Martínez Menchén, «La tortuga y Aquiles », Inquisidores, Madrid, ZERO ZYX, 1977, p. 179.

15. "Te dedicas a recuperar tu memoria, individual y coral, contra la obligada amnesia del vencido en la guerra civil " suggère Manuel Vázquez Montalbán à Juan Marsé qu’il qualifie ainsi que lui-même- de "memoriones memoriadores " "La memoria de Juan Marsé " dans José Belmonte Serrano et José Manuel López de Abiada, Nuevas tardes con Marsé, Murcia, Nausicaä, 2002, p. 278-279.

16. Juan Marsé, Un día volveré, Barcelona, Plaza y Janés, 1982, p. 287.

17. Jean Tena, "Lécriture de la mémoire : la génération innocente " dans Annie BussièrePerrin, Le roman espagnol actuel (1975-2000). Pratique d'écriture, Tome II, Montpellier, CERS, 2001, p. 237-274.

18. Carmen Martín Gaite, El cuento de nunca acabar, Madrid, Trieste, 1983, p. 390. Quant à José Manuel Caballero Bonald, il évoque "los fantasmas circulares de la memoria" ("Los aventis de Marsé "-1989-, Copias del natural, Madrid, Alfaguara, 1999, p. 226).

19. Juan Marsé, Si te dicen que caí (1973), Barcelona, Seix Barral, 1976, p. 13. 
Selon José Manuel Caballero Bonald, «de todos los componentes del grupo narrativo de los años 50, Juan Marsé es -para mi gusto- el que con más saludable y solvente capacidad indagatoria (cinematográfica, iba a decir) ha escrito la novela de su generación ${ }^{20}$. Dans les deux autoportraits que l'on vient de commenter, Juan Marsé, avec l'efficacité qui le caractérise, a encore produit, pour reprendre l'épigraphe de Carlos Barral, « un texto de reconocimiento generacional ». 